\title{
Time-resolved assessment of single-cell protein secretion by sequencing
}

\author{
Tongjin $\mathbf{W u}^{1}$, Howard John Womersley²,
}

Jiehao Wang ${ }^{3}$, Jonathan Adam Scolnick ${ }^{3}$, Lih Feng Cheow ${ }^{1,2, *}$

${ }^{1}$ Department of Biomedical Engineering, Faculty of Engineering, National University

of Singapore, Singapore 117583, Singapore.

${ }^{2}$ Institute for Health Innovation and Technology, National University of Singapore,

Singapore 117599, Singapore.

${ }^{3}$ Proteona Pte. Ltd., Singapore 117525, Singapore.

*Correspondence: lihfeng.cheow@nus.edu.sg 


\section{SUMMARY}

Secreted proteins play critical roles in cellular communication and functional orchestration. Methods enabling concurrent measurement of cellular protein secretion, phenotypes and transcriptomes are still unavailable. Here, we describe time-resolved assessment of protein secretion from single cells by sequencing (TRAPS-seq). Released proteins are trapped onto cell surface via affinity matrices, and the captured analytes together with phenotypic markers can be probed by oligonucleotide-barcoded antibodies and simultaneously sequenced with transcriptomes. We used TRAPS-seq to interrogate secretion dynamics of pleiotropic cytokines (IFN- $\gamma$, IL-2 and TNF- $\alpha$ ) of early activated human $\mathrm{T}$ lymphocytes, unraveling limited correlation between cytokine secretion and its transcript abundance with regard to timing and strength. We found that early central memory $T$ cells with CD45RA expression (TCMRA) are the most effective responders in multiple cytokine secretion, and polyfunctionality involves unique yet dynamic combinations of gene signatures over time. TRAPS-seq presents a useful tool for cellular indexing of secretions, phenotypes, and transcriptomes at single-cell resolution.

\section{INTRODUCTION}

Secreted proteins, such as cytokines, chemokines, cytotoxic molecules, antibodies, and growth factors, make up the secretome and play crucial roles in mediating cell-cell communication and shaping functionality under physiological and pathological conditions ${ }^{1-5}$. Correlation between functional protein release (e.g., cytokines and chemokines) and cellular phenotypes has been extensively studied yet is still inconclusive, especially for immune cells recently undergoing activation/inflammation processes $^{6,7}$. The reasons according for this may include the heterogeneity of cell subpopulations and variable dynamics of protein secretion with regard to timing and strength ${ }^{8-10}$. Moreover, protein secretion is not always consistent with its transcript level that 
could result from the collective effects of epigenetic ${ }^{11}$ and/or translational/posttranslational regulation ${ }^{12-15}$. Therefore, approaches enabling simultaneous measurement of multiple cellular features (e.g., protein secretion, phenotypic traits, and transcriptional profiles) at single-cell resolution, and ultimately being able to achieve time-resolved information of single-cell protein release, will be important not only for the understanding of cell biology but also for the rational design of disease treatment regimens.

Classical methods such as enzyme-linked immunosorbent assay (ELISA) and enzyme-linked immunospot (ELISpot) assess the population-level capacity of protein secretion by measuring extracellularly released analytes without information of source cells producing them ${ }^{16}$. Flow cytometry-assisted intracellular staining assay (ICS) is useful to evaluate single-cell protein expression, which however generally requires trapping of secreted proteins inside the cells prior to fixation/permeabilization at the time point measured, thus unable to represent the true cell secretion and temporal secretion dynamics, also precluding further analysis when live cells matter. Some derivatives of these techniques such as Flow-FISH (combined analysis of cellular protein via ICS and mRNA by probing) and FluoroSpot (fluorescencebased multicolor ELISpot) are still less feasible to determine the temporal dynamics of single-cell protein secretion ${ }^{17,18}$. To meet these gaps, platforms using micro-droplets ${ }^{19-22}$ or microengraved arrays ${ }^{8,10,23}$ are the methods of choice, in which cells are individually compartmentalized and the released proteins are captured onto capture antibodies-coated solid surface (e.g., bead or array slide). In addition, secretion capture assay (SCA), an approach to capturing secreted analytes onto cell surface using affinity matrices such as bispecific antibody that binds to both cell-surface protein and secreted protein, has long been established for the characterization of cytokine-secreting $T$ cells and antibody-expressing $B$ cells ${ }^{24-26}$. While these techniques make it feasible 
to determine multiplexed and dynamic protein secretion from live single cells with known phenotypes $8,10,21$, they are inevitably hindered by the numbers of secretion targets and surface markers as well that can be concurrently labelled for deep cell characterization due to fluorescence spectral overlap. Moreover, there are limited flexibility and throughput to recover the individual cells of interest for downstream single-cell RNA sequencing (scRNA-seq) even with the possibility using index sorting 27,28 .

scRNA-seq has provided biological insights in a diverse range of research areas and becomes even more powerful with concurrent measurement of multiplexed cell-surface markers using oligonucleotide-barcoded detection antibodies (Ab-Oligo $)^{29-31}$. Inspired by these advances and inadequacies, we describe time-resolved assessment of protein secretion from single cells by sequencing (TRAPS-seq), a method that relies on the time-dependent trapping of secreted proteins on the cell surface and the use of Ab-Oligo barcoding for the quantification of differential protein secretion, enabling cellular indexing of secretion dynamics, cell-surface phenotypic traits, and RNA profiles from sequencing-based readout.

\section{RESULTS}

\section{Design rationale of the TRAPS-seq}

To make protein secretion addressable to its source cell, we took advantage of the design of secretion capture assay (SCA), in which secreted proteins of interest were captured onto the cell surface via affinity matrices such as bispecific antibody that binds to both cell-surface protein and secreted protein (Figure 1A). To enable measurement of protein secretion via sequencing-based readout across thousands of single cells at a time, we adopt oligonucleotidebarcoded detection antibodies (Ab-Oligo) (Figure 1B) that can be easily integrated into current platforms for single-cell multi-omics studies. 
112 The selection of cell-surface anchor sites for cytokine-capturing antibodies

113 (CapAbs) has profound effects on downstream biological studies. CD45, a

114 stable and abundant pan-leukocyte surface glycoprotein is an ideal choice for

115 CapAbs anchoring (Figure S1A). We showed that CD45 staining has negligible

116 effects on T cell expansion capability (Figure S1B). We further demonstrated

117 that the abundance of cell-surface CD45 is not a limiting factor to generation of

118 affinity matrix for multiplexed cytokine-capturing assay (Figure S1C, D).

119 Immediately after the capture of secreted proteins, cells are simultaneously

120 labeled with multiplexed sequencing-compatible Ab-Oligos targeting the

121 surface-bound secreted proteins (Figure S2 and Figure S3) and cell-surface

122 markers (Figure 1C). Subsequent high-throughput single-cell sequencing

123 enables integrated analysis of single cell transcriptome, secretion and

124 phenotypes (Figure 1D). 
A

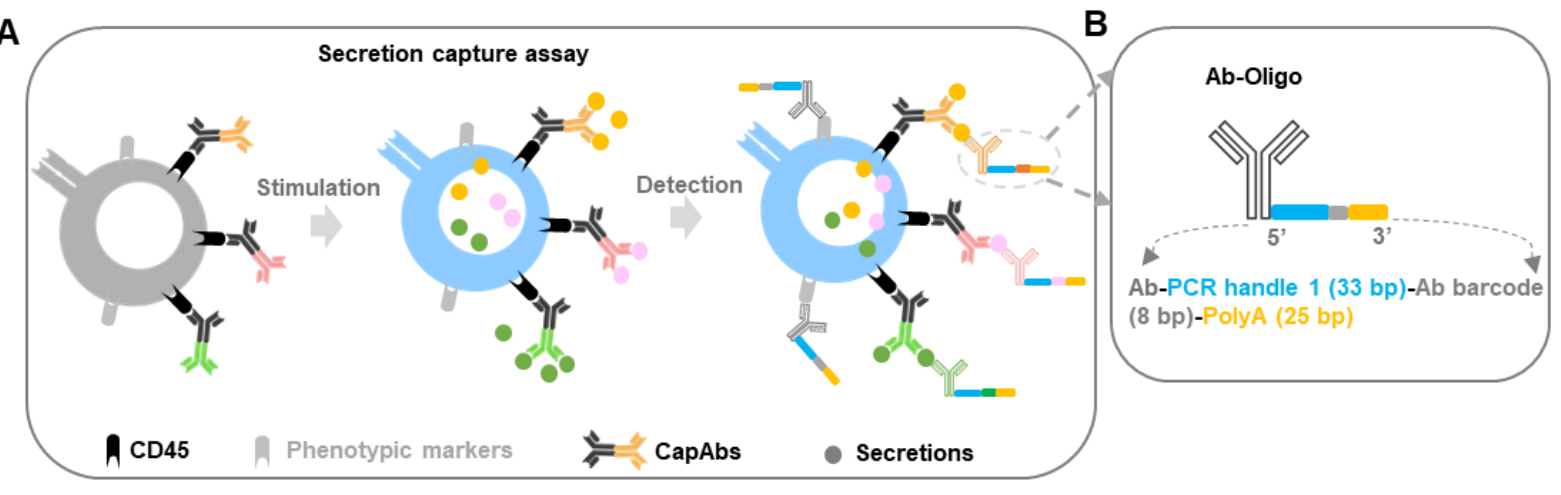

C

Integrated sequencing of protein secretion, phenotypic traits, and transcriptomic profiles

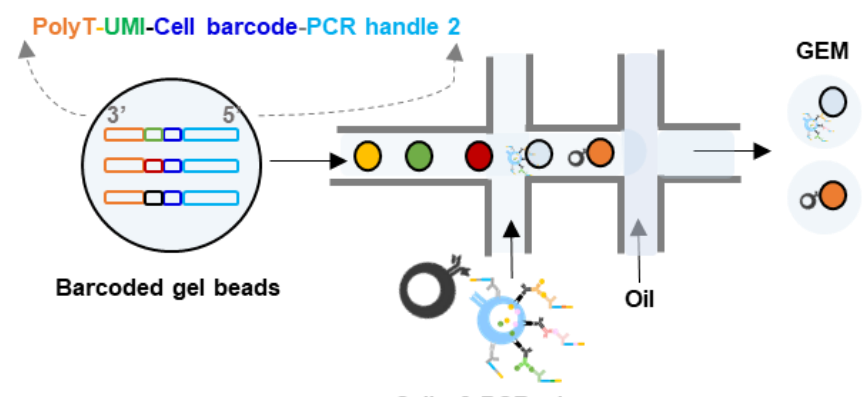

Cells \& PCR mix

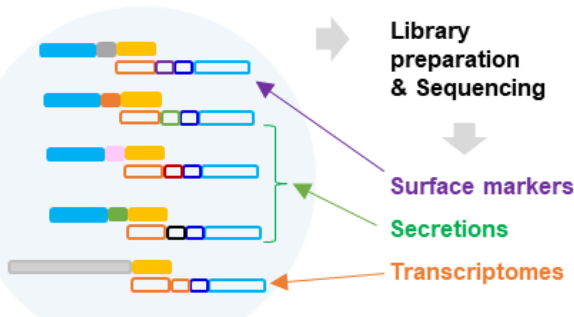

Cell lysis \& RT

D

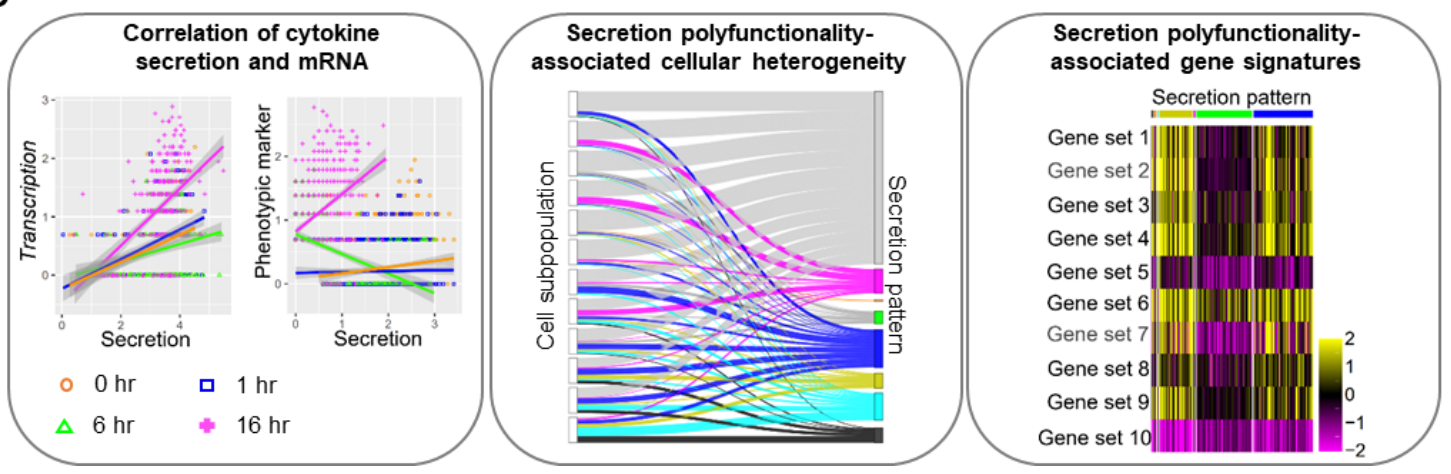

141 Figure 1. Schematic representation of TRAPS-seq workflow

(A-B) Cells were pre-coated with cytokine-capturing matrices (CapAbs) for standard

144 (Ab-Oligo) that may target either the captured cytokines or cell surface markers (B).

145 (C) The stained cells were subjected to droplet-based cell-bead encapsulation and sequencing libraries construction that include mRNA content and barcodes 
analysis reveals relationship between cytokine secretion, phenotypic traits and transcription state.

\section{TRAPS-seq enables multimodal single-cell characterization}

Pleiotropic cytokines (IFN- - , IL-2 and TNF- $\alpha$ ) are key factors orchestrating antigen-elicited immune responses and have been extensively studied ${ }^{1,2}$. However, there is still a lack of our understanding about their secretion dynamics with regard to timing and strength, and also the underling cellular and molecular signatures at a higher dimension.

Herein, we used TRAPS-seq to interrogate the secretion dynamics and functionality of human lymphocytes activated by anti-CD3/CD28 microbeads (Figure 2A), a TCR-dependent stimulation approach used to mimic physiological activation of T cells. Cytokine secretion at time points of 1 hour, 6 hours and 16 hours post cell activation was measured (Figure 2A and Figure S4A). These timepoints were selected to overlap the known expression timing of pleiotropic cytokines (IFN- $\gamma$, IL-2 and TNF- $\alpha$ ) by $T$ cells ${ }^{8,32}$. The single-cell transcriptomes of unmodified and CapAbs modified lymphocytes was indistinguishable, implying that CapAbs precoating did not perturb cellular transcriptomes (Figure S4B). Activation-induced enhancement or repression of protein secretion was observed for all three cytokines (Figure S4C), suggesting TRAPS-seq was capable of measuring the heterogeneity of protein secretion 
170 at the single-cell level. Cells were clustered according to their expression

171 profiles of phenotypic protein markers (Figure S4D) and annotated by using

172 both surface-protein expression and a panel of genes associated with T-cell

173 activation/differentiation (Figure S4E; refer to Figure S6A about background

174 subtraction). Notably, differentiated T-cell subsets and NK-cell subpopulations

175 are clustered more distinctly by surface markers (Figure S5), demonstrating

176 that multimodal analysis of single cells allowed better cell characterization

177 beyond transcriptome alone (Figure S5) ${ }^{33}$.

We found that there was limited correlation between cytokine secretion and

180 gene expression (refer to Figure S6B, C about background subtraction) with regard to timing and strength (Figure 2B-E and Figure S7), and this inconsistency was also true for the surface proteins detected (Figure S7B) ${ }^{29,34}$.

TNF- $\alpha$ and IL-2 secretion occurred earlier than IFN- $\gamma$ following cell activation

(Figure 2C, D). For long-term activation (6-16 hours), the percentage of IL-2positive cells remained relatively stable while expression level of IL2 mRNA continuously increased. On the other hand, both the proportion of cells and gene expression levels of cells secreting TNF- $\alpha$ or IFN- $\gamma$ increased over time

188 (Figure 2D). TNF- $\alpha$ or IL-2 secretion was higher than IFN- $\gamma$ in response to initial stimulation (1 hour) but diminished at late activation stages (6-16 hours), while IFN-y secretion per cell gradually increased (Figure 2E). This is in line with the

191 gradual functionality acquisition of T/NK cells upon activation and 
192 differentiation $9,35,36$. These data suggest that time-resolved measurement of

193 both protein secretion and transcription state is more insightful in our

194 understanding of cellular function acquisition.

196 A deep mapping of cytokine mRNA and protein onto cell subtypes revealed high

197 diversity of cells in cytokine production (Figure 2F-H). This heterogeneity was

198 cell phenotypes and/or activation state-related, resulting in discordance in both

199 the timing and multiplicity between cytokine gene generation and protein

200 release (Figure 2I, J). Intriguingly, NK cells had a sparse secretion of IL-2 post

201 initial activation and shifted to IFN-Y during late stage, the gene expression,

202 however, possessed a distinct pattern from IFNG to IFNG ${ }^{+}$IL2 $^{+}$(Figure 2G, H).

203 The presence of IL2 mRNA in NK cells is controversial but could be culture- 
A

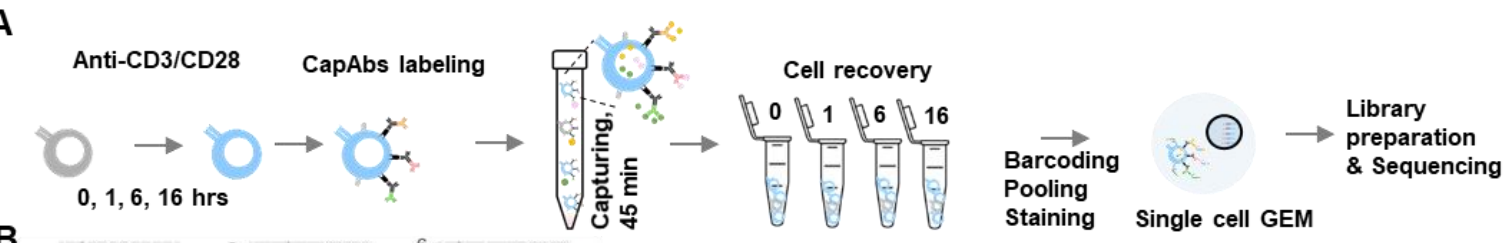

B
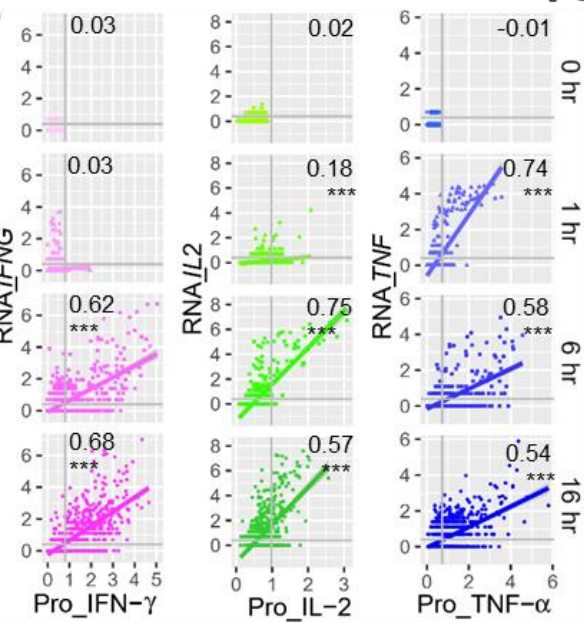

$\mathbf{F}$

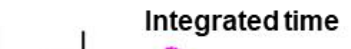

C

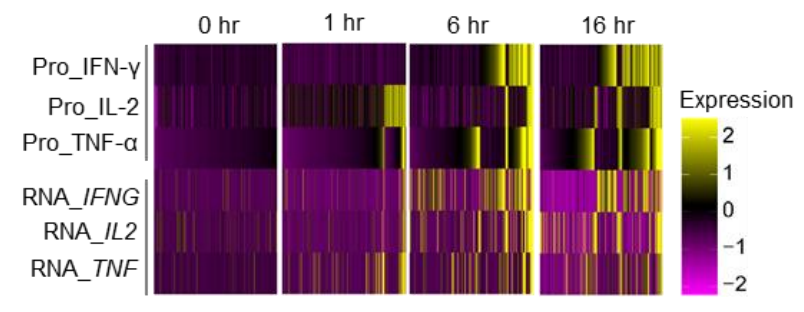

D

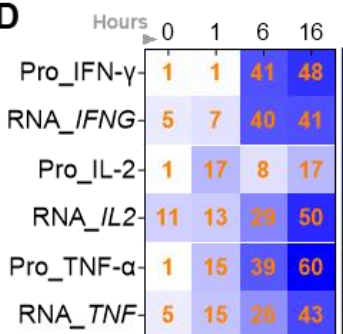

E

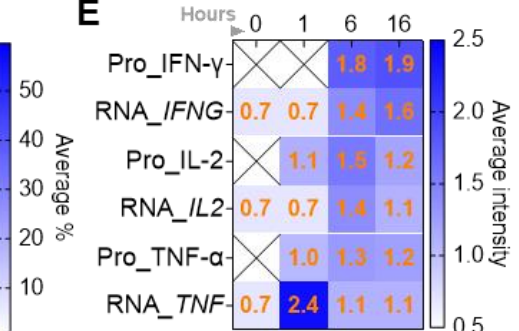

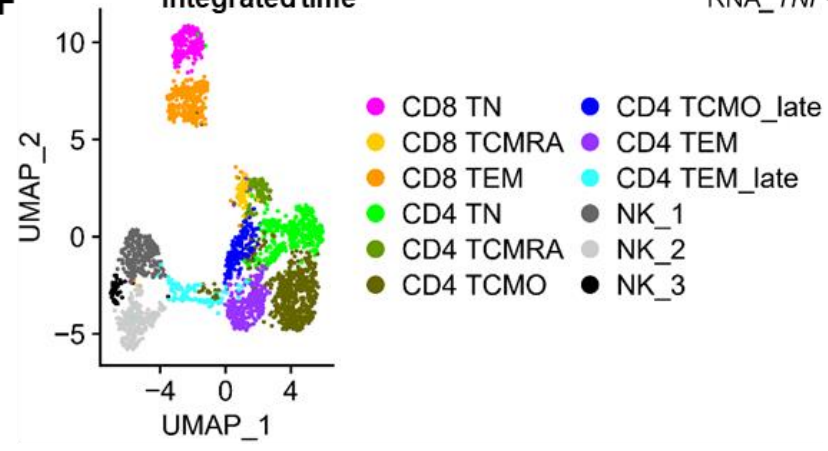

G

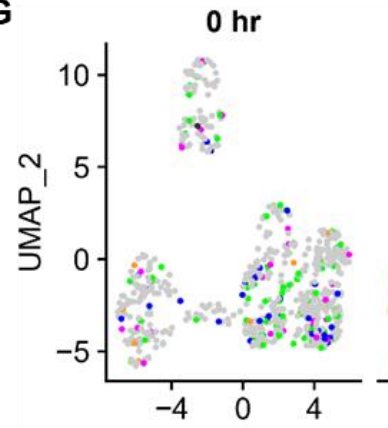

$1 \mathrm{hr}$

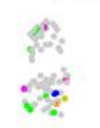

$x$
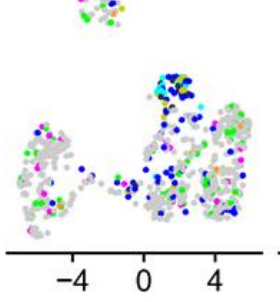

UMAP
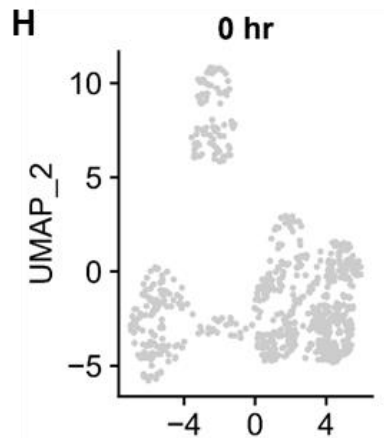

$1 \mathrm{hr}$

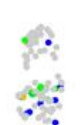

I

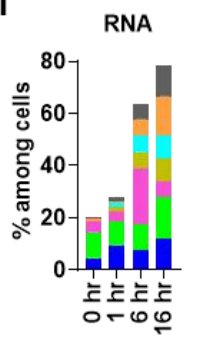

$\mathbf{J}$

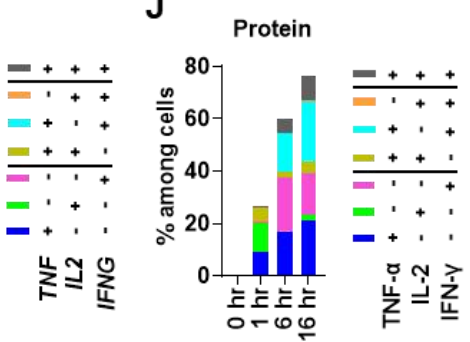

$16 \mathrm{hr}$

$\%$

30

Tri-positive

- IFNG+IL2

- IFNG+TNF

IL2+TNF

- IFNG

- IL2

- TNF

Negative

$16 \mathrm{hr}$

$6 \mathrm{hr}$

8

- Tri-positive

- IFN- $\gamma+$ IL-2

- IFN $-\gamma+$ TNF $-\alpha$

- IL-2+TNF- $\alpha$

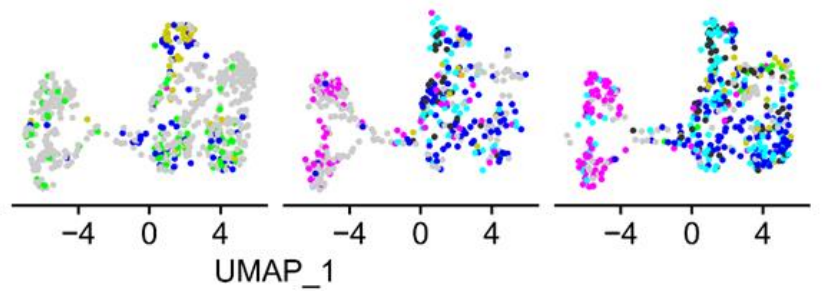

- IFN- $\gamma$

- IL-2

- TNF- $\alpha$

Negative 
Figure 2. TRAPS-seq enables integrated profiling of single-cell protein secretion, phenotypes and transcriptomes

(A) Experimental design of TRAPS-seq using human lymphocytes (B cells-depleted) activated by anti-CD3/CD28 microbeads for indicated time points. (B-C) Dynamic change of cytokine secretion versus transcript level as shown by scatter plots. Shown are log-normalized values and Pearson's correlation coefficient with significance test. ${ }^{*} P<.05,{ }^{* *} P<.01,{ }^{* * *} P<.001(B)$

(B) or heatmap at single-cell level over time (C). (D-E)

Shown were summary of cell percentile with cytokine releasing or cytokine gene expression and strength, correspondingly. $(\mathrm{F}-\mathrm{H})$ Unsupervised cell clustering based on the expression abundance of phenotypic markers (F). The annotated cells were colored by their cytokine gene expression $(G)$ or cytokine release profile $(H)$. Shown histogram plots were summary of cytokine expression polyfunctionality corresponding to $G(I)$ and $H(J)$.

TRAPS-seq identifies cell subpopulations with heterogeneous secretion with protective immunity ${ }^{39,40}$ and favorable therapeutic outcomes ${ }^{41}$. A comprehensive understanding of the occurrence and maintenance of these heterogeneous cells at both cellular and molecular levels would be invaluable. 

memory T cells with CD45RA expression (TCMRA) (Figure S4E) ${ }^{42,43}$ regardless

231 of $\mathrm{CD}^{+}{ }^{+}$or $\mathrm{CD}^{+} \mathrm{T}$ cells had the rapidest cytokine secretion, primarily TNF- $\alpha^{+}$

$232 \mathrm{IL}^{-} 2^{+}$and $\mathrm{TNF}-\mathrm{\alpha}^{+}$within one hour post activation, while those moredifferentiated counterparts like central memory without CD45RA expression ( $\left.\mathrm{T}_{\mathrm{CMO}}\right)$ and effector memory ( $\mathrm{T}_{\mathrm{EM}}$ and $\mathrm{TEM}_{\mathrm{EM}}$ late $)$ tended to secret mono-cytokine such as TNF- $\alpha^{+}$or IL-2 during initial stimulation (Figure $3 \mathrm{~A}$ ). $\mathrm{CD}^{+}$TCMRA continued to release multiple cytokines, specifically IFN- $\gamma$-involved triple or double positive cytokines, whereas amongst $\mathrm{CD}^{+} \mathrm{T}$ cells, CD4 ${ }^{+} \mathrm{T}_{\mathrm{CMRA}}$ and $\mathrm{CD}^{+}{ }^{+} \mathrm{T}_{\mathrm{CMO}}$ late were the multi-cytokine releasing cells (Figure $3 \mathrm{~B}, \mathrm{C}$ ). This discrepancy could result from activation-coupled subtler phenotypic change from $\mathrm{CD}^{+}{ }^{+} \mathrm{T}_{\text {CMRA }}$ to $\mathrm{CD} 4^{+} \mathrm{T}_{\mathrm{CMO}} / \mathrm{CD}^{+}{ }^{+} \mathrm{T}_{\mathrm{CMO}}$ late during stimulation ${ }^{39}$. On the other hand, CD8 ${ }^{+} \mathrm{T}_{\mathrm{EM}}$ and $\mathrm{NK}$ cells had mainly contributed to the mono-IFN-Y expression per cell during late activation stage (Figure 3B, C). Naïve T cells $\left(T_{N}\right)$

243 instead were shown to be more inert to CD3/CD28-engaged activation but endowed with the highest capacity in secreting various combination of cytokines for a long term stimulation (Figure 3C), consistent with their developmental diversity post exposed to exogenous antigens ${ }^{44}$.

While there was limited correlation between individual cytokine secretion and surface marker expression (Figure S8), combinatory analysis of surface phenotypic/activating markers could partially identify cells of distinct secretion 
251 potency (Figure 3D-F). T cells with triple cytokine release at 1 hour post

252 stimulation had stem-cell memory (Tscm)-like phenotypic markers (e.g., CCR7,

253 CD45RA, CD27, CD28 and CD95) ${ }^{43}$ (Figure 3D). Late activating T cells with

254 triple cytokine release additionally had high abundance of the activation marker

255 CD69 and degranulation molecule CD107a (Figure 3E, F). Cells capable of

256 secreting IL-2 ${ }^{+}$TNF- $\alpha^{+}$were characterized by high CD107a and to a less extent

257 CD95 in their resting/early state, and both of which were diminished along with

258 the elevation of CD69 for a long-term activation, however, cells producing IFN-

$259 \mathrm{Y}^{+}$TNF- $\alpha^{+}$were characteristic of the opposite dynamics (Figure 3D-F).

260 Therefore, cytokine secretion potency of CD3/CD28-engaged T cells was cell

261 subtype associated and affected by their dynamic change of

262 phenotypic/activating markers, but less correlated to change of a singular

marker. 
A

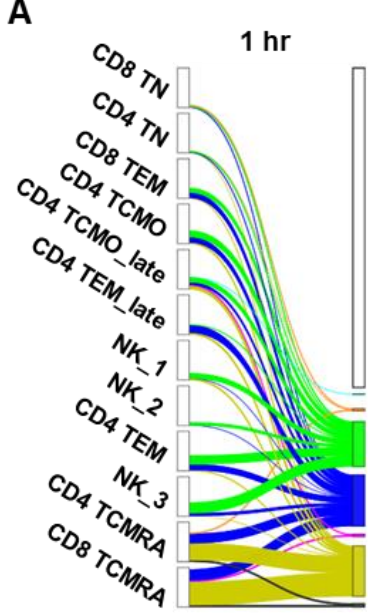

D

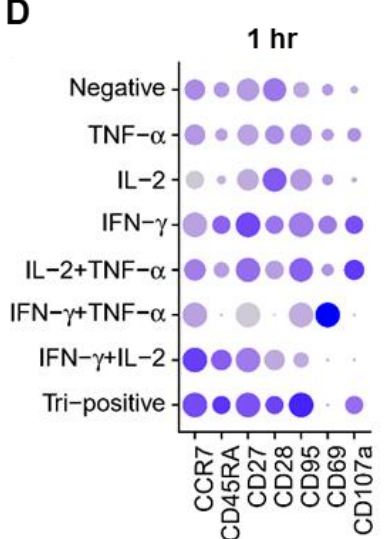

B

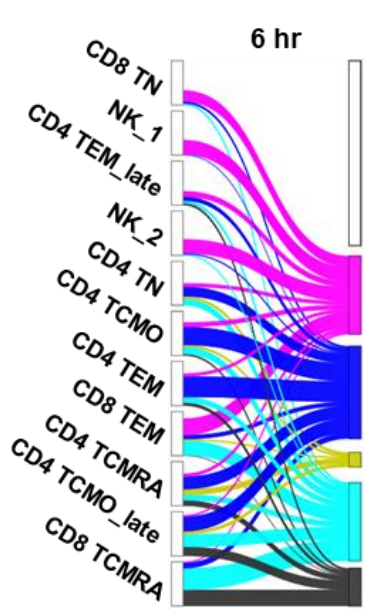

E

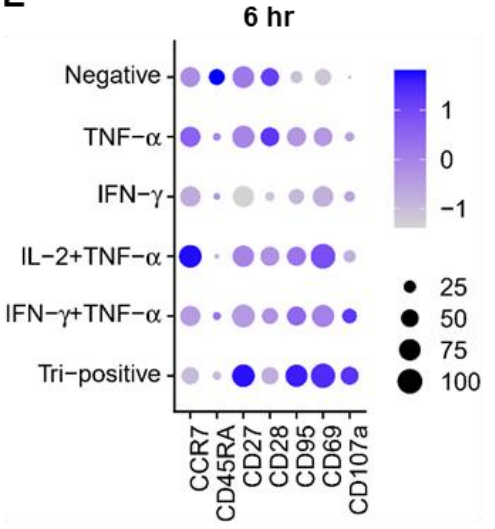

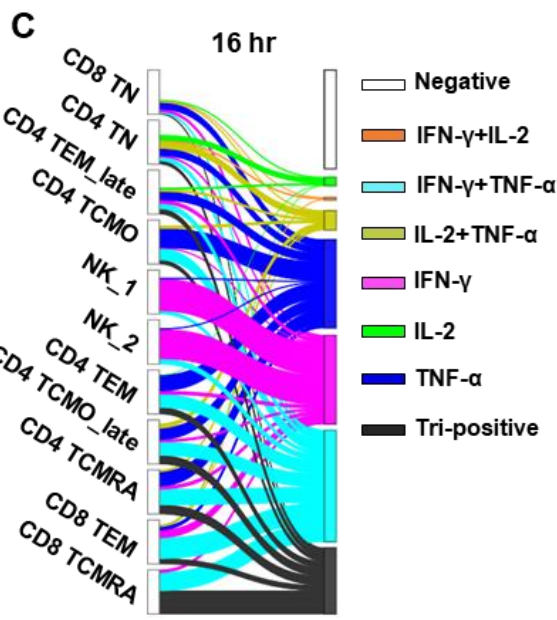

$\mathbf{F}$

Figure 3. TRAPS-seq identifies $T_{C M R A}$ as the most effective responders in multiple

(A-C) Sankey diagrams depict how cell composition/activation state change over time may affect their cytokine secretion profiles. Cell numbers for each cell type were normalized. (D-F) Expression of a panel of phenotypic markers in T cells with indicated cytokine release profile. 
used for the study of polyfunctional T cells, however, being with a lack of real cytokine secretion. We next attempted to characterize the transcriptional signatures that may be correlated with cytokine secretion potency.

Notably, despite limited correlation between cytokine secretion and mRNA abundance (Figure 2B, C and Figure S7), IFNG, IL2, and TNF were still among the top genes that differentiated cells with or without that cytokine secretion, correspondingly (Figure 4A, B). FOS (AP-1 transcription factor subunit), EGR1 (early growth response protein 1) and $C D 69$ (early activation marker) were enriched in cells secreting IL-2 $2^{+} \mathrm{TNF}-\alpha^{+}$and to a lesser extent TNF- $\alpha^{+}$only, but not IL-2 $2^{+}$(Figure 4A), consistent with the finding that IL-2 $2^{+} \mathrm{TNF}-\alpha^{+} / \mathrm{TNF}-\alpha^{+}$was among the first wave of stably released cytokines during T-cell early activation (Figure 3A). Cells with IFN- $\gamma$-involved cytokine release were distinguishable from those without IFN-y secretion, manifested by an upregulation of IFNG but also genes encoding chemokine ligands (e.g., CCL4, CCL3, CCL4L2, XCL2, and CCL5) and cytotoxic molecules (e.g., GZMB and NKG7) (Figure 4A, B), indicating their strong effector function. Cells with triple cytokine secretion had a general downregulation of chemotactic and cytotoxic mRNAs (e.g., CCL4, CCL3, CCL4L2 NKG7, GZMB and GNLY) compared to IFN- $\gamma^{+}$or IFN- $\gamma^{+}$TNF$\alpha^{+}$cells (Figure 4B). However, triple cytokine positive cells shared some unique features with those secreting early cytokines IL-2 $2^{+} \mathrm{TNF}-\alpha^{+} / \mathrm{TNF}-\alpha^{+}$, for example, an upregulation of genes relating to immune and inflammatory responses such 

(G1/S-specific cyclin-D3) and EZH2 (enhancer of zeste homolog 2, critical for epigenetic modification) (Figure 4A, B), suggesting an unique state of this triple cytokine-secreting cell subpopulation which was endowed with not only immediate/regulatory functions but also superior commitment to ensuing proliferation/differentiation ${ }^{39,45}$.

To depict the whole landscape of T-cell cytokine secretion and transcriptome profile, we calculated gene set-based scores at single-cell level using the curated hallmark gene sets from the Molecular Signatures Database (MSigDB).

We found that IL-2 and TNF- $\alpha$ signaling were highly engaged in IL-2/TNF- $\alpha-$ involved polyfunctional cells but not those secreting IL-2 or TNF- $\alpha$ only, implying that effective acquisition of multi-cytokine secretion could involve on both IL-2and TNF- $\alpha$-mediated paracrine/autocrine effects (Figure S9A-C). On the other hand, IFN- $\gamma$ signaling was more engaged in cells secreting TNF- $\alpha$ only,

314 suggesting potential regulation on TNF- $\alpha$ secretion via IFN- $\gamma-$ mediated paracrine effects (Figure S9A, D). Moreover, cells with triple cytokine secretion had a generally higher enrichment of genes involved in oncogenic transcription

317 factor Myc-regulated targets and oxidative phosphorylation (OXPHOS), but a 
318 downregulation of genes relating to IFN-a response, apoptosis and hypoxia

319 (Figure 4C and Figure S9E), a favorable cellular state for optimal cytokine

320 secretion and survival. Of note, despite that the expression timing and strength

321 of genes representative of known signatures could be dynamically varied over

322 time, it was still feasible to characterize cells of discernable secretion potency

by a combination of certain unique gene set-based signatures for each

324 individual time points (Firue S9F-H). 
bioRxiv preprint doi: https://doi.org/101101/2021.12 19.473400; this version posted December 21, 2021. The copyright holder for this preprint (which was not certified by peer review) is the author/funder, who has granted bioRxiv a license to display the preprint in perpetuity. It is made available under aCC-BY-NC-ND 4.0 International license.

A

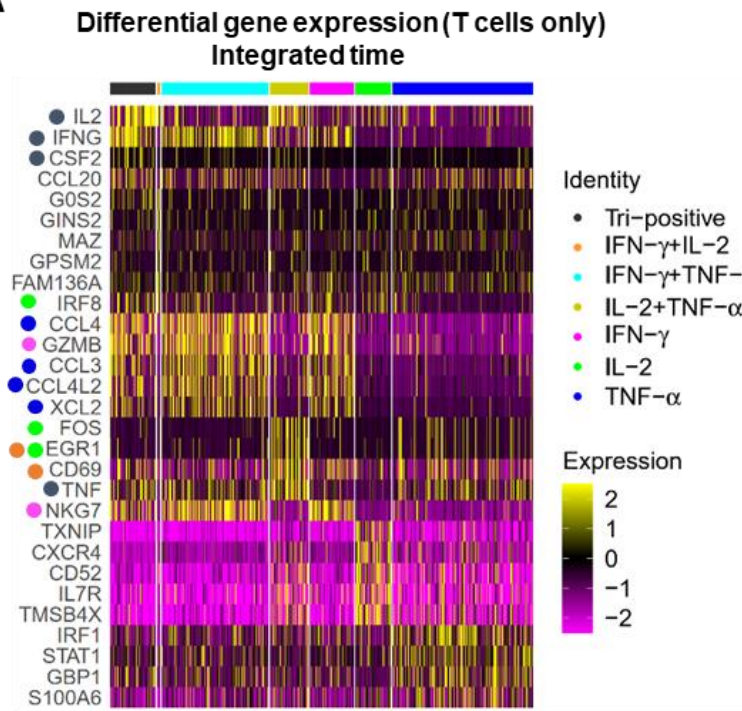

B Differential gene expression( $T$ cells only)

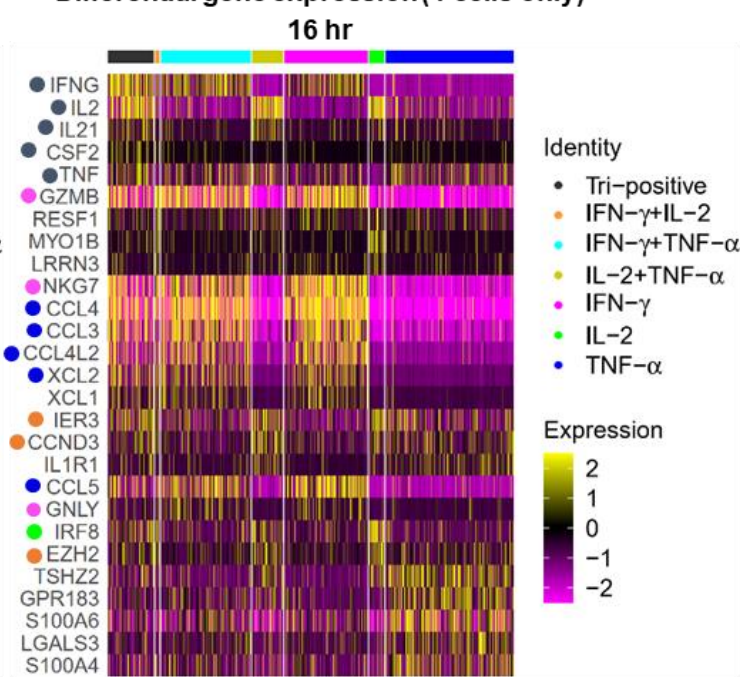

- Cytokine Cytotoxic factor $\bullet$ Chemokine $\bullet$ Transcription factor Differentiation/mitosis

C

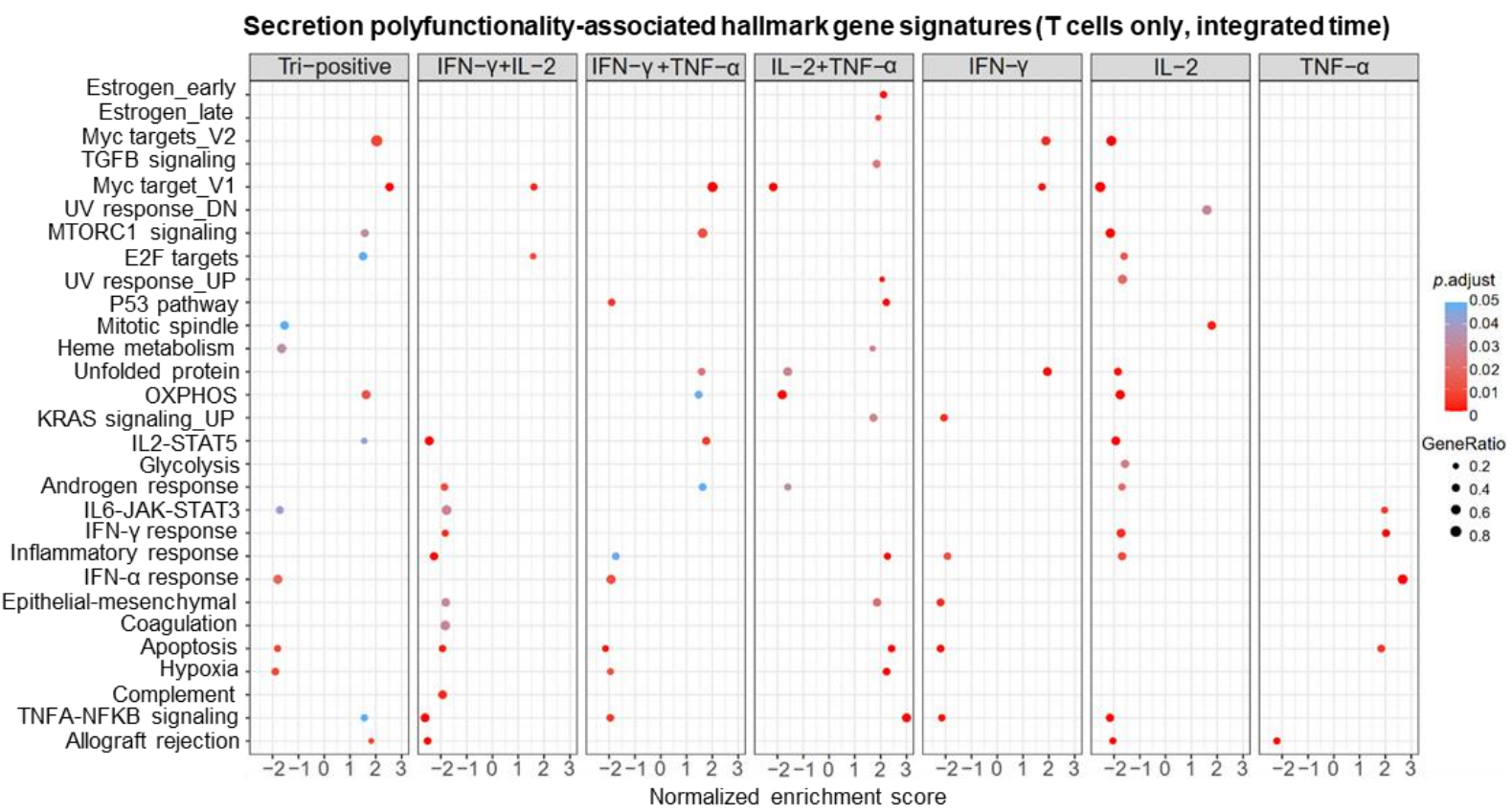

Figure 4. Cytokine-secreting polyfunctionality-associated differential gene-set

327 (A-B) Differential gene expression of $T$ cells with indicated cytokine release. Shown 


\section{DISCUSSION}

333 In this study, we describe for the first time a sequencing-based approach to

334 measuring dynamic protein secretion at single-cell level (TRAPS-seq). We have

335 also demonstrated how TRAPS-seq can be simultaneously integrated with

336 sequencing of transcriptomes and phenotypic proteins to dissolve the cellular

337 and molecular heterogeneity of differential cytokine secretion.

We found that there was limited correlation between cytokine protein secretion and mRNA expression for all cytokines detected (IFN- $\gamma$, IL-2 and TNF- $\alpha$ ) in CD3/CD28-engaged $T$ cells. Combined analysis of single-cell intracellular cytokine abundance and mRNA level (Flow-FISH), however, showed that the expression dynamics of intracellular cytokine protein was generally consistent with its $\mathrm{mRNA}^{18}$. This discrepancy could mainly result from the measurement of cytokine protein expression by TRAPS-seq is a snapshot compared to progressive accumulation (with the protein transporter machinery inhibited) by

347 Flow-FISH at the time point measured. The disconnect of cytokine secretion and transcription has profound effects on protective and dysfunctional immune responses (e.g., autoimmune diseases and inhibited antitumor activity) ${ }^{14,15,46}$. TRAPS-seq, when combined with single-cell transcriptome measurement, would provide us with more comprehensive information to advance our understanding of cytokine-involved physiological and pathological processes. 
TCMRA cells are phenotypically resembling Tscm (or Tscm-like) ${ }^{43,47}$, characterized

by positive expression of canonical naïve/memory-specific markers CCR7 and

CD45RA (but lower than $\mathrm{T}_{\mathrm{N}}$ ), and high expression of Fas receptor CD95 and

cell persistence-related costimulatory molecule CD27. TCMRA has also a highly

presented lysosomal-associated membrane protein-1 (LAMP-1/CD107a),

suggesting a potential state of TCMRA (especially CD8 ${ }^{+} \mathrm{T}_{\mathrm{CMRA}}$ ) ready for

immediate cytotoxic function. We have also demonstrated that TCMRA was the

most effective responder in secreting ample amount of IL-2 and TNF- $\alpha$ or TNF-

$\alpha$ only soon post CD3/CD28 engagement and towards the secretion of triple

IFN- $\mathrm{Y}^{+}$had a dramatic upregulation of genes involved in IL2-STAT and TNF-

NFKB signaling pathways, and genes favoring cell survival and

proliferation/differentiation (e.g., CSF2, IER3, CCND3, EZH2, and Myc-

367 regulated genes). These data have extended our previous finding that the generation of TCMRA during the production of therapeutic $\mathrm{T}$ cells was wellcorrelated with the proportion of $\mathrm{T}_{\mathrm{N}}$ in cellular starting material ${ }^{42}$, and cell products containing more TCMRA were manifested by younger phenotypes and

371 superior functions that are recognized to be good for therapeutic outcomes.

372 Thus, combined analysis of secretion-included single-cell multi-omics would see their versatility in the characterization of secretion profiles and dynamics

374 that could be associated with protective immunity or harmful inflammation, 

Cluster) for cell sorting. near future.

\section{ACKNOWLEDGEMENTS}

facilitating the rational design of therapeutic regimens ${ }^{7,41,48}$.

Finally, despite its usefulness of TRAPS-seq, a limitation of this strategy could be cytokine capture multiplexing. To circumvent this, additional common anchoring sites of CapAbs could be further explored beyond CD45 (used in this study), for example, via chemical modification of cell surface to increase artificial binding sites ${ }^{49}$. Meanwhile, DNA barcode design with enhanced signalto-noise ratio would also be helpful ${ }^{50}$. Undoubtedly, TRAPS-seq has filled up a gap in the area of single-cell multi-omics studies ${ }^{51}$, making cytokine secretion indexable to multi-modalities of its source cell, and would find its versatility in

\section{SUPPLEMENTARY INFORMATION}

Supplementary information is available for this manuscript.

This work was supported by funding from Singapore Ministry of Education Academic Research Fund Tier 2 (MOE-000063) and Institute for Health Innovation and Technology (iHealthtech), NUS. The authors acknowledge technical support from the Flow Cytometry Laboratory (NUS Medical Sciences 


\section{AUTHOR CONTRIBUTIONS}

T.W and L.F.C conceived and designed the study. T.W performed the experiments and data analysis with input from L.F.C and J.A.S in data mining and assistances from H.J.W and J.W in sequencing techniques. T.W and L.F.C wrote the manuscript with feedback from J.A.S. All authors approved the submission.

\section{DECLARATION OF INTERESTS}

L.F.C and T.W. are listed as co-inventors on a patent application related to this work (SG Patent Application No. 10202112484W). J.A.S is a scientific cofounder of Proteona Pte. Ltd., a medicine company providing the services of single-cell multi-omics to advance cancer therapy. J.W is an employee of Proteona Pte. Ltd.

\section{METHODS}

\section{Cells and reagents}

Apheresis blood samples from healthy donors were gifts from the Health Sciences Authority (HSA), Singapore, with approval from Institutional Review Board and informed consent from donors (NUS-IRB no. H-18-038E). Ficoll Paque Plus (GE Healthcare, 17-1440-02) was used for peripheral blood mononuclear cells (PBMC) separation. RPMI1640 medium (ThermoFisher, A1049101) with $10 \%$ fetal bovine serum (FBS) (ThermoFisher, 10270106) was 
used in cell culture. Antibodies and Ab-Oligo used in this work were listed in synthesized by IDT (Integrated DNA Technologies) and listed in Table S2.

Cytofix/Cytoperm reagent kit (BD Biosciences, 554714) was used for

(Biolegend, 422301) was used to block Fc receptor-introduced non-specific

staining. Phosphate buffered saline (PBS) with 2\% FBS was used for staining buffer and $0.5 \%$ FBS for washing buffer, except where specified.

\section{Ab-Oligo conjugates generation and validation}

Ab-Streptavidin conjugates were generated according to the instruction of

Lightning-Link Streptavidin Antibody Labeling Kit (Novus Biologicals, 708-0030).

Excess free oligo-biotin was removed by streptavidin-coated microbeads

0.5\% FBS, $1 \mathrm{mM}$ EDTA, and $0.09 \% \mathrm{NaN}_{3}$.

Unstimulated PBMCs were stained with cytokine-capturing CapAb and incubated in cytokine-containing culture supernatant at $4^{\circ} \mathrm{C}$ for $20 \mathrm{~min}$. For 
washing the cells were further stained by indicated Ab-Oligo $(0.3-0.5 \mu \mathrm{g} / \mathrm{test})$ for 20 min. Meanwhile, the competitively blocking function of modified Ab-Oligo conjugates was validated by their capability to reduce the binding of fluorescent antibodies (Ab-Fluo) of the same antibody clones.

\section{Cytokine secretion capture assay (SCA)}

Cytokine SCA was carried out according to the standardized protocol with the kits (Miltenyi Biotec) as listed in Table. S1, but with slight modification. Briefly, $\mathrm{CD}_{19}{ }^{+}$cells-depleted lymphocytes were sorted in Moflo Astrios cell sorted (Beckman Coulter) and kept in incubator overnight before stimulation. Cells were activated by anti-CD3/CD28 Dynabeads (Gibco, 11161D) at 2:1 bead-tocell ratio for indicated time periods in 96-well U-bottom plate. Post beads removal, cells were stained with saturated cytokine-capturing antibodies for 15 min at $4{ }^{\circ} \mathrm{C}$ and subjected to secretion capture at $37^{\circ} \mathrm{C}$ for 45 min with gentle rotation. The captured cytokines can be detected by fluorescent antibodies for flow cytometry analysis or by Ab-Oligo for downstream sequencing experiments.

\section{Sample staining with pooled Ab-Oligo}

461 Ab-Oligo for cell surface markers $(1 \mu \mathrm{g} / \mathrm{test})$ and captured cytokines (0.3-0.5 $\mu \mathrm{g} / \mathrm{test}$ ) were pooled and filtered by Amicon Ultra- 0.5 centrifugal column 

re-suspended in $50 \mu \mathrm{l}$ PBS with $2 \%$ heat-inactivated FBS. Cells for each time points $(0,1,6,16$ hours $)$ were individually labelled with $\beta 2 \mathrm{M}$-targeting hashtags sand Fc-blocking agents, while indicated, control cells without CapAb modification were labelled with CD45-targeting hashtags instead. These five group of cells were pooled together after washing and stained with Ab-Oligo cocktail for 45 min at $4{ }^{\circ} \mathrm{C}$. Cells were washed with cold PBS containing $2 \%$ FBS for 4 times and re-suspended in PBS before run on the Chromium Controller (10x Genomics).

\section{Sequencing libraries preparation for TRAPS-seq}

The 10x Genomics single cell 3' reagent kits (v3.1 Chemistry) was used to generate single-cell barcoded initial sequencing libraries following reverse transcription that include complementary DNA (CDNA) of mRNA and protein

477 libraries representative of cytokines, surface markers and sample-indicating hashtags. Post cleanup using silane beads, $35 \mu \mathrm{l}$ of the eluted DNA sample was pre-amplified for the $1^{\text {st }}$-round following the standard protocol with the addition of two additive primers for surface protein and hashtag (listed in Table S2) and cycled as follows: as follows: $98^{\circ} \mathrm{C} 3 \mathrm{~min}, 12$ cycles of: $98^{\circ} \mathrm{C} 15 \mathrm{~s}, 63^{\circ} \mathrm{C}$ $20 \mathrm{~s}$, and $72^{\circ} \mathrm{C} 1 \mathrm{~min}$; Then an extension step of $1 \mathrm{~min}$ at $72^{\circ} \mathrm{C}$. The $1^{\text {st }}$-round 
library.

The amplified cDNA product was subjected to final gene expression library construction according to the instructions with the kit. Part of the protein DNA product $(5 \mu \mathrm{l})$ was used for the preparation of sequencing libraries corresponding to surface marker and cytokine while additional part $(5 \mu \mathrm{l})$ was used for the construction of sequencing library of sample hashtags using KAPA HiFi Master Mix (Roche). The $2^{\text {nd }}$-round P7/read 2-incorporated PCR primers used for protein library preparation were listed in Table S2 with the P5/read 1incorporated primer the same to that used in cDNA library. The size range and concentration of final library constructions was verified by Agilent 2100 Bioanalyzer.

\section{Single-cell RNA and protein data processing}

The libraries for cDNA and sample hashtag were pooled and sequenced in Illumina HiSeq platform by Novogene. The protein libraries for surface protein and cytokine were sequenced in MiSeq platform using MiSeq Reagent Kit v3 and customized sequencing primers (listed in Table S2). Raw cDNA FASTQ data was processed using Cell Ranger v4.0 (10x Genomics) with default parameters. A total of 4,899 cell barcodes with 90,834 reads/cell were reported. These cDNA library-derived cell barcodes were used to match the unique molecular identifier (UMI) counts of sample hashtags, surface protein and 
cytokine using CITE-seq-Count with a Hamming distance of 1 for both cell barcodes calling and UMI counting ${ }^{52}$. Pre-processed count matrices were

509 imported into Seurat package (v4.0) $)^{33}$, and dead cells were removed by filtering

510 out cells expressing $>5 \%$ mitochondrial transcript counts. Post cell

511 demultiplexing, a total of 4,010 singlets were remained for downstream analysis.

512 For the analysis of cytokine secretion, where indicated, sparse dots above 99

513 quantiles of the background-counts distribution at "0 hr" (Figure S6C) and

514 apparently discrete dots within data of other time points (Figure S7A) were 515 removed.

\section{Gene set enrichment analysis (GSEA)}

518 Differential gene expression by Wilcoxon Rank Sum test between cells of

519 distinct secretion pattern were performed in Seurat (v4.0) using sctransformnormalized gene expression counts $^{33,53}$, and generated gene lists were

521 imported for GSEA using R programs embedded in the clusterProfiler through

522 the use of curated hallmark gene sets from the Molecular Signatures Database 523 (MSigDB, v7.4) $)^{54,55}$. Normalized enrichment scores (NES) and adjusted $P$ 524 value $(<0.05)$ were ranked for presentation.

\section{Single-cell gene set-based signatures' calculation}

527 Single-Cell Signature Explorer was used to score gene set-based signature for each individual cells ${ }^{56}$. Briefly, sctransform-normalized gene expression matrix 
corresponding to each individual time points was imported into the Single-Cell Signature Scorer to compute signature score per cell over a list of hallmark gene sets from MSigDB (v7.4). Dimensionality reduction plot corresponding to each individual time point was produced by UMAP based on the cytokine secretion measured. The calculated signature scores for individual cells were visualized on the UMAP plot via Single-Cell Signature Viewer. Differentially expressed gene-set signatures (average logFC $>0.25$; adjusted $P<0.05 ; 20 \%$ of cells within the cluster expressing the target gene) across cell groups with different secretion patterns were performed by Seurat (v4.0) using a Wilcoxon Rank Sum test and shown by heatmap plots ${ }^{33}$.

\section{Pearson correlation coefficient test}

To make it quantifiable and comparable between protein and mRNA expression or protein and protein expression, the varied background signaling of surface marker and cytokine staining was subtracted (refers to Figure S6A, C). For the Pearson's correlation analysis, where indicated, either all cells or cells that had at least one positive expression of protein or mRNA were included. Paired Pearson's correlation between protein and mRNA or cytokine release and surface protein marker was performed using correlation package ${ }^{57}$.

\section{Data analysis}

Flow cytometry data were acquired in CytoFLEX (Beckman Coulter) and 


\section{REFERENCES}

5591 Kumar, B. V., Connors, T. J. \& Farber, D. L. Human T Cell Development, Localization, and

$560 \quad$ Function throughout Life. Immunity 48, 202-213, doi:10.1016/j.immuni.2018.01.007 (2018).

5612 Grivennikov, S. I., Greten, F. R. \& Karin, M. Immunity, inflammation, and cancer. Cell 140, 883-899, doi:10.1016/j.cell.2010.01.025 (2010).

5633 Dranoff, G. Cytokines in cancer pathogenesis and cancer therapy. Nature reviews. Cancer 4, 1122, doi:10.1038/nrc1252 (2004).

$5654 \quad$ Uhlen, M. et al. The human secretome. Sci Signal 12, doi:10.1126/scisignal.aaz0274 (2019).

5665 Antonioli, L., Blandizzi, C., Pacher, P., Guilliams, M. \& Hasko, G. Rethinking Communication in the Immune System: The Quorum Sensing Concept. Trends Immunol 40, 88-97, doi:10.1016/j.it.2018.12.002 (2019).

6 Appay, V., van Lier, R. A., Sallusto, F. \& Roederer, M. Phenotype and function of human T lymphocyte subsets: consensus and issues. Cytometry. Part A : the journal of the International Society for Analytical Cytology 73, 975-983, doi:10.1002/cyto.a.20643 (2008).

Varadarajan, N. et al. A high-throughput single-cell analysis of human CD8(+) T cell functions reveals discordance for cytokine secretion and cytolysis. J Clin Invest 121, 4322-4331, doi:10.1172/JCI58653 (2011).

Han, Q. et al. Polyfunctional responses by human T cells result from sequential release of cytokines. Proceedings of the National Academy of Sciences of the United States of America 109, 1607-1612, doi:10.1073/pnas.1117194109 (2012).

Salerno, F., Paolini, N. A., Stark, R., von Lindern, M. \& Wolkers, M. C. Distinct PKC-mediated posttranscriptional events set cytokine production kinetics in CD8(+) T cells. Proceedings of the National Academy of Sciences of the United States of America 114, 9677-9682, doi:10.1073/pnas.1704227114 (2017). functional heterogeneity in response to pathogenic ligands. Proceedings of the National Academy of Sciences of the United States of America 112, E607-615, doi:10.1073/pnas.1416756112 (2015).

11 Michalak, E. M., Burr, M. L., Bannister, A. J. \& Dawson, M. A. The roles of DNA, RNA and 
histone methylation in ageing and cancer. Nat Rev Mol Cell Biol 20, 573-589, doi:10.1038/s41580-019-0143-1 (2019).

Liu, Y., Beyer, A. \& Aebersold, R. On the Dependency of Cellular Protein Levels on mRNA Abundance. Cell 165, 535-550, doi:10.1016/j.cell.2016.03.014 (2016).

3 Turner, M. \& Diaz-Munoz, M. D. RNA-binding proteins control gene expression and cell fate in the immune system. Nature immunology 19, 120-129, doi:10.1038/s41590-017-0028-4 (2018).

14 Salerno, F. et al. Translational repression of pre-formed cytokine-encoding mRNA prevents chronic activation of memory T cells. Nature immunology 19, 828-837, doi:10.1038/s41590018-0155-6 (2018).

15 Villarino, A. V. et al. Posttranscriptional silencing of effector cytokine mRNA underlies the anergic phenotype of self-reactive $\mathrm{T}$ cells. Immunity 34, 50-60, doi:10.1016/j.immuni.2010.12.014 (2011).

16 Favre, N., Bordmann, G. \& Rudin, W. Comparison of cytokine measurements using ELISA, ELISPOT and semi-quantitative RT-PCR. Journal of immunological methods 204, 57-66, doi:10.1016/s0022-1759(97)00033-1 (1997).

17 Gazagne, A. et al. A Fluorospot assay to detect single T lymphocytes simultaneously producing multiple cytokines. Journal of immunological methods 283, 91-98, doi:10.1016/j.jim.2003.08.013 (2003).

18 Nicolet, B. P., Guislain, A. \& Wolkers, M. C. Combined Single-Cell Measurement of Cytokine mRNA and Protein Identifies T Cells with Persistent Effector Function. Journal of immunology 198, 962-970, doi:10.4049/jimmunol.1601531 (2017).

19 Mazutis, L. et al. Single-cell analysis and sorting using droplet-based microfluidics. Nature protocols 8, 870-891, doi:10.1038/nprot.2013.046 (2013).

20 Konry, T., Golberg, A. \& Yarmush, M. Live single cell functional phenotyping in droplet nanoliter reactors. Scientific reports 3, 3179, doi:10.1038/srep03179 (2013).

21 Bounab, Y. et al. Dynamic single-cell phenotyping of immune cells using the microfluidic platform DropMap. Nature protocols 15, 2920-2955, doi:10.1038/s41596-020-0354-0 (2020).

Eyer, K. et al. Single-cell deep phenotyping of IgG-secreting cells for high-resolution immune monitoring. Nature biotechnology 35, 977-982, doi:10.1038/nbt.3964 (2017).

23 Love, J. C., Ronan, J. L., Grotenbreg, G. M., van der Veen, A. G. \& Ploegh, H. L. A microengraving method for rapid selection of single cells producing antigen-specific antibodies. Nature biotechnology 24, 703-707, doi:10.1038/nbt1210 (2006).

24 Manz, R., Assenmacher, M., Pfluger, E., Miltenyi, S. \& Radbruch, A. Analysis and sorting of live cells according to secreted molecules, relocated to a cell-surface affinity matrix. Proceedings of the National Academy of Sciences of the United States of America 92, 19211925 (1995).

25 Burel, J. G., Apte, S. H., Groves, P. L., McCarthy, J. S. \& Doolan, D. L. Polyfunctional and IFN-gamma monofunctional human CD4(+) T cell populations are molecularly distinct. JCI Insight 2, e87499, doi:10.1172/jci.insight.87499 (2017).

26 Pinder, C. L. et al. Isolation and Characterization of Antigen-Specific Plasmablasts Using a Novel Flow Cytometry-Based Ig Capture Assay. Journal of immunology 199, 4180-4188, doi:10.4049/jimmunol.1701253 (2017).

27 Schulte, R. et al. Index sorting resolves heterogeneous murine hematopoietic stem cell 
populations. Exp Hematol 43, 803-811, doi:10.1016/j.exphem.2015.05.006 (2015).

28 Baron, C. S. et al. Cell Type Purification by Single-Cell Transcriptome-Trained Sorting. Cell 179, 527-542 e519, doi:10.1016/j.cell.2019.08.006 (2019).

29 Stoeckius, M. et al. Simultaneous epitope and transcriptome measurement in single cells. Nature methods 14, 865, doi:10.1038/nmeth.4380 (2017).

30 Peterson, V. M. et al. Multiplexed quantification of proteins and transcripts in single cells. Nature biotechnology 35, 936-939, doi:10.1038/nbt.3973 (2017).

31 Shahi, P., Kim, S. C., Haliburton, J. R., Gartner, Z. J. \& Abate, A. R. Abseq: Ultrahighthroughput single cell protein profiling with droplet microfluidic barcoding. Scientific reports 7, 44447, doi:10.1038/srep44447 (2017).

32 Friedl, P. \& Gunzer, M. Interaction of T cells with APCs: the serial encounter model. Trends Immunol 22, 187-191, doi:10.1016/s1471-4906(01)01869-5 (2001).

33 Hao, Y. et al. Integrated analysis of multimodal single-cell data. Cell 184, 3573-3587 e3529, doi:10.1016/j.cell.2021.04.048 (2021).

34 Nicolet, B. P. \& Wolkers, M. C. Limited but gene-class specific correlation of mRNA and protein expression in human CD8(+) T cells. bioRxiv, 2020.2004.2021.053884, doi:10.1101/2020.04.21.053884 \%J bioRxiv (2020).

35 Gattinoni, L. et al. Acquisition of full effector function in vitro paradoxically impairs the in vivo antitumor efficacy of adoptively transferred CD8+ T cells. J Clin Invest 115, 1616-1626, doi:10.1172/JCI24480 (2005).

He, X. S. et al. T cell-dependent production of IFN-gamma by NK cells in response to influenza A virus. J Clin Invest 114, 1812-1819, doi:10.1172/JCI22797 (2004).

37 Vitolo, D. et al. Rapid Il-2-induced adherence of human natural killer cells. Expression of mRNA for cytokines and IL-2 receptors in adherent NK cells. Journal of immunology 151, 1926-1937 (1993).

38 Jokhi, P. P., King, A., Sharkey, A. M., Smith, S. K. \& Loke, Y. W. Screening for cytokine messenger ribonucleic acids in purified human decidual lymphocyte populations by the reversetranscriptase polymerase chain reaction. Journal of immunology 153, 4427-4435 (1994).

39 Seder, R. A., Darrah, P. A. \& Roederer, M. T-cell quality in memory and protection: implications for vaccine design. Nature reviews. Immunology 8, 247-258, doi:10.1038/nri2274 (2008).

40 Panagioti, E., Klenerman, P., Lee, L. N., van der Burg, S. H. \& Arens, R. Features of Effective T Cell-Inducing Vaccines against Chronic Viral Infections. Frontiers in immunology 9, 276, doi:10.3389/fimmu.2018.00276 (2018).

1 Rossi, J. et al. Preinfusion polyfunctional anti-CD19 chimeric antigen receptor T cells are associated with clinical outcomes in NHL. Blood 132, 804-814, doi:10.1182/blood-2018-01828343 (2018).

2 Wu, T., Luah, Y. H., Luo, Y., Womersley, H. J. \& Cheow, L. F. Label-free lymphocytes reconstitution using side scatter for optimal $\mathrm{T}$ cell manufacturing. bioRxiv, 2020.2011.2009.375584, doi:10.1101/2020.11.09.375584 (2020).

3 Fuertes Marraco, S. A. et al. Long-lasting stem cell-like memory CD8+ T cells with a naivelike profile upon yellow fever vaccination. Science translational medicine 7, 282ra248, doi:10.1126/scitranslmed.aaa3700 (2015).

van den Broek, T., Borghans, J. A. M. \& van Wijk, F. The full spectrum of human naive T cells. Nat. Rev. Immunol. 18, 363-373, doi:10.1038/s41577-018-0001-y (2018). 
67545 Westerhof, L. M. et al. Multifunctional cytokine production reveals functional superiority of

676 memory CD4 T cells. European journal of immunology 49, 2019-2029, 677 doi:10.1002/eji.201848026 (2019).

67846 Salerno, F. et al. Critical role of post-transcriptional regulation for IFN-gamma in tumor-

679 infiltrating T cells. Oncoimmunology 8, e1532762, doi:10.1080/2162402X.2018.1532762 $680 \quad$ (2019).

68147 Gattinoni, L. et al. A human memory T cell subset with stem cell-like properties. Nature 682 medicine 17, 1290-1297, doi:10.1038/nm.2446 (2011).

68348 Fajgenbaum, D. C. \& June, C. H. Cytokine Storm. N Engl J Med 383, 2255-2273, 684 doi:10.1056/NEJMra2026131 (2020).

68549 Kellam, B., De Bank, P. A. \& Shakesheff, K. M. Chemical modification of mammalian cell $686 \quad$ surfaces. Chem Soc Rev 32, 327-337, doi:10.1039/b211643j (2003).

$68750 \quad$ Ke, Y., Ong, L. L., Shih, W. M. \& Yin, P. Three-dimensional structures self-assembled from 688 DNA bricks. Science 338, 1177-1183, doi:10.1126/science.1227268 (2012).

690 Chappell, L., Russell, A. J. C. \& Voet, T. Single-Cell (Multi)omics Technologies. Annu Rev Genomics Hum Genet 19, 15-41, doi:10.1146/annurev-genom-091416-035324 (2018). Roelli, P., Flynn, B. \& Gui, G. Hoohm/CITE-seq-Count: 1.4 .2 (Version 1.4.2). Zenodo, doi:10.5281/zenodo.2590196 (2019). data using regularized negative binomial regression. Genome biology 20, 296, doi:10.1186/s13059-019-1874-1 (2019). 